\title{
CAP-EOM-CCSD method with smooth Voronoi CAP for metastable electronic states in molecular clusters
}

\author{
James R. Gayvert and Ksenia B. Bravaya* \\ Department of Chemistry, Boston University \\ Boston, Massachusetts 02215, USA
}

\begin{abstract}
The complex absorbing potential (CAP) approach offers a practical tool for characterization of energies and lifetimes of metastable electronic states, such as temporary anions and core ionized states. Here, we present an implementation of the smooth Voronoi CAP combined with equation-of-motion coupled cluster with single and double substitutions method for metastable states. The performance of the smooth Voronoi and a standard box CAPs is compared for different classes of systems: resonances in isolated molecules and in molecular clusters. The results of the benchmark calculations indicate that the choice of the CAP shape should be guided by the character of the metastable states. While Voronoi CAPs yield stable results in the case of a resonance localized on one molecule, their performance in the cases of states delocalized over two or more molecular species can deteriorate due to the CAP leaking into the vacuum region between the moieties.
\end{abstract}

\section{INTRODUCTION}

Electronic resonances are metastable electronic states with finite lifetimes lying in the ionization/detachment continuum. Common examples include transient anions formed by electron attachment, and core-excited and core-ionized states that can undergo Auger decay or related relaxation pathways. These states are key players in a wide variety of processes ranging from those that occur in high energy environments (plasmonic photocatalysis, attosecond and X-ray spectroscopies) to low energy electron-molecule scattering (DNA damage from secondary electrons, interstellar chemistry) [1-6]. Resonances belong to the continuous spectrum of the electronic Hamiltonian, so they are not part of the usual Hilbert space

\footnotetext{
*Electronic address: bravaya@bu.edu
} 
of square integrable functions [7]. Theoretical description of these states is generally not possible by means of conventional quantum chemistry methods developed for bound states, and one has to use special techniques to obtain accurate resonance energies and lifetimes.

Non-Hermitian quantum mechanics (NHQM) techniques provide an appealing approach that allows one to leverage existing quantum chemistry methodology to treat metastable electronic states [8]. In NHQM formalisms, a resonance appears as a single square-integrable eigenstate of a non-Hermitian Hamiltonian. The resonance parameters can be extracted from the corresponding complex eigenvalue:

$$
E=E_{R}-\frac{i \Gamma}{2}
$$

where the real part of the energy $\left(\mathrm{E}_{R}\right)$ is the resonance position, and the imaginary part (- i $\Gamma / 2)$ is the half-width, which is inversely proportional to the lifetime of the metastable state $\left(\tau=\frac{\hbar}{\Gamma}\right)[7,9]$. One of the NHQM formalisms that has been successfully used to study resonances in molecular systems is the complex absorbing potential (CAP) method. CAPs are imaginary potentials added to the Hamiltonian, originally devised as a numerical technique to absorb outgoing wave packets near the boundaries of finite grids [10]. CAPs have also been applied in the time-independent framework to study various problems, including the Stark effect, reactive scattering, and quantum transport in molecular devices [11-13], and are now routinely used for evaluation of metastable states parameters [14-20].

In this work, we explored the effects of the CAP shape on computed resonance parameters in molecular systems. In the context of electronic structure of metastable electronic states, CAPs are used to transform the resonance into a single square integrable state, and to render the state accessible by means of standard bound-state techniques [9]. To this end, the electronic Hamiltonian is augmented with an imaginary potential $(-i \eta W)$

$$
H_{C A P}(\eta)=H-i \eta W
$$

where $\eta$ is the CAP strength parameter, and $\mathrm{W}$ is a real potential which vanishes in the vicinity of the molecular system and grows with distance [9]. An ideal CAP must satisfy the following two criteria. First, it should not perturb the system in the inner, molecular region, i.e. it should not introduce reflections. Secondly, the CAP should absorb the outgoing tail of the resonance wave function $[9,16]$. Several approaches for minimizing these residual reflections have been proposed. Examples include "reflection-free" and "transformative" 
CAPs [21-23]. In most practical applications, simple quadratic CAPs such as those discussed here are used, as they can easily be combined with existing electronic structure theory codes.

As the CAP-augmented Hamiltonian depends on the strength of the CAP (Eq. 2), a choice has to be made on the optimal value of $\eta$ which provides the best estimate of the resonance position and width. In a complete one-electron basis, the exact resonance position and width are obtained in the limit of an infinitesimally weak CAP $\left(\eta \rightarrow 0^{+}\right)$[9]. In practice when finite bases are used, an optimal CAP strength $\eta_{\text {opt }}$ is found by locating a stationary point on the eigenvalue trajectory $E(\eta)$, for example using the minimum of the logarithmic velocity criterion $\left(\left|\eta \frac{d E}{d \eta}\right| \rightarrow \min \right)[9]$. The shape of this trajectory, and the best estimate of resonance position and width for a given basis set/method depends on the choice of the CAP.

The most commonly used form of the CAP for electronic structure calculations is the quadratic "box CAP", which is defined by three cutoff parameters $\left(R_{x}^{0}, R_{y}^{0}, R_{z}^{0}\right)$ which specify the onset of the box in each Cartesian coordinate [14, 24].

$$
\begin{array}{r}
W=W_{x}+W_{y}+W_{z} \\
W_{\alpha}=\left\{\begin{array}{cc}
0 & \left|r_{\alpha}\right|<R_{\alpha}^{0} \\
\left(r_{\alpha}-R_{\alpha}^{0}\right)^{2} & \left|r_{\alpha}\right|>R_{\alpha}^{0}
\end{array}\right\}
\end{array}
$$

where $\alpha=x, y$, or $z$.

Practical recipes for choosing the box size have been suggested, and correction schemes have been shown to the reduce the dependence of the results on the box size [25, 26]. However, the rigid box-like shape is not always reflective of the detailed geometry of a molecular system, and it can be difficult to apply this type of CAP to systems which do not efficiently fill the space.

The idea of defining a CAP using each atom's Voronoi cell was proposed by Sommerfeld and Ehara [16, 27]. The Voronoi absorbing potential wraps uniformly around any molecule, and is specified by a single cutoff radius $r_{c u t}$. Following the work by Sommmerfeld and Ehara $[16,27]$ we use the "smooth Voronoi" potential, which smooths out the edges between Voronoi cells, making the resulting CAP more amenable to numerical integration [16]. In 
this case, the CAP is defined as follows [16]:

$$
W(\mathbf{r})=\left\{\begin{array}{cl}
0 & r_{W A} \leq r_{c u t} \\
\left(r_{W A}(\mathbf{r})-r_{c u t}\right)^{2} & r_{W A}>r_{c u t}
\end{array}\right\}
$$

$r_{W A}(\mathbf{r})$ in Eq. 4 is the effective distance to the molecular system evaluated as a weighted average of the distances to all nuclei:

$$
r_{W A}(\mathbf{r})=\sqrt{\frac{\sum_{i} w_{i}\left|\mathbf{r}-\mathbf{R}_{i}\right|^{2}}{\sum_{i} w_{i}}}
$$

The weights are defined as follows:

$$
w_{i}=\frac{1}{\left(\left|\mathbf{r}-\mathbf{R}_{i}\right|^{2}-r_{\min }^{2}+1 b o h r^{2}\right)^{2}}
$$

where $r_{\min }$ is the distance to the closest atom: $r_{\min }=\min _{i}\left|\mathbf{r}-\mathbf{R}_{i}\right|$.

In contrast to the box CAP, the Voronoi CAP shares exactly the symmetry of the molecular system, and is flexible, i.e. is easily adjustable to changing nuclear configurations, for example in dynamical simulation. Importantly, unlike the box CAP, the Voronoi CAP can leak into the empty spaces in molecular clusters as it wraps around each molecule. Thus, one can expect differences in behavior of these two types of CAP for different types of systems.

To shed light on these differences, we have implemented the smooth Voronoi CAP in the Q-Chem program package [28] and compared its performance to that of the standard box CAP for three types of systems: resonances in diatomic molecules $\left(\mathrm{N}_{2}^{-}, \mathrm{CO}^{-}\right)$, localized resonances in a model cluster $\left(\mathrm{N}_{2}^{-}\left(\mathrm{H}_{2} \mathrm{O}\right)_{2}\right)$, and delocalized resonance states in the anion of the experimentally observed [29-31] carbon monoxide dimer $\left((C O)_{2}^{-}\right)$. We employed the CAP equation-of-motion coupled cluster method with single and double substitutions for electron attachment CAP-EOM-EA-CCSD $[14,25,26]$ to compute the resonance energies and widths of all model systems.

The structure of the manuscript is as follows. We outline the main features of the CAP method in Sec. II A and discuss the technical details in Sec. II B. The performance of box and smooth Voronoi CAPs for description of resonance parameters in diatomics, localized and delocalized resonances in clusters is discussed in Secs. III A, III B, and III C, respectively. 


\section{METHODS}

\section{A. Complex Absorbing Potentials}

In the CAP method, resonance parameters are obtained as complex eigenvalues (Eq. 1) of the CAP-augmented electronic Hamiltonian (Eq. 2). The CAP-augmented Hamiltonian is complex symmetric $\left(H(\eta)^{\dagger}=H(\eta)^{*}\right)$, and so the usual Hermitian inner product is replaced with the c-product $[9,32]$

$$
\left(\phi_{i}(\mathbf{r}) \mid \phi_{j}(\mathbf{r})\right)=\int d \mathbf{r} \phi_{i}(\mathbf{r}) \phi_{j}(\mathbf{r})
$$

In a finite basis representation, an optimal $\eta_{\text {opt }}$ which provides the best estimate of resonance position and width must be identified. The $\eta_{\text {opt }}$ is highly system dependent, and varies with the electronic structure method, basis set, and the form of the CAP used. To search for $\eta_{\text {opt }}$, the calculation is repeated over a range of $\eta$ values, generating complex eigenvalue trajectories which depend on $\eta$. When using the logarithmic velocity criterion [9], $\eta_{\text {opt }}$ corresponds to the CAP strength on the eigenvalue trajectory where $\left|\eta \frac{d E}{d \eta}\right|$ has its minimum.

Results obtained in this fashion are rather sensitive to the CAP onset, due to unphysical perturbations of the resonance wave function induced by the CAP [25]. A few correction schemes have been proposed to improve the accuracy and stability of CAP augmented calculations [25, 33]. Here we employ the scheme proposed by Jagau et al. [25], which is based on first order "de-perturbation" of the real $\left(E^{R}\right)$ and imaginary $\left(E^{I}\right)$ parts of the energy.

$$
\begin{aligned}
U^{R} & =E^{R}-\eta \operatorname{Tr}\left[W \gamma^{I}\right] \\
U^{I} & =E^{I}+\eta \operatorname{Tr}\left[W \gamma^{R}\right]
\end{aligned}
$$

where $W$ in the equation above is CAP $W$ matrix (Eq. 2), and $\gamma_{R} / \gamma_{I}$ are the real and imaginary parts of the state reduced one particle density matrix. Resonance parameters are obtained by locating stationary points of the first-order corrected eigenvalues $U^{R}$ and $U^{I}$ (Eq. 7) with respect to $\eta$. This approach has been shown to significantly reduce the dependence of the resonance parameters on the CAP onset compared to those obtained from uncorrected trajectories $[25,26]$. In this work, we follow the protocol of Jagau et al [25], in which the reported corrected energies and widths are obtained from the earliest extrema 
of $U^{R}$ and $U^{I}$ which display approximate stationary behavior. Representative corrected $\eta$-trajectories for each of the states are given in the SI.

\section{B. Computational Details}

We have implemented the smooth Voronoi CAP in the Q-Chem program package, and the method was released as part of Q-Chem [28] version 5.2.2. A locally modified version of Q-Chem 5.1.1 was used for these calculations. All complex eigenvalues were obtained using the CAP-EOM-EA-CCSD method [14, 25, 26].

The basis sets used in our calculations consist of Dunning's correlation consistent ccpVTZ [34] and aug-cc-pVTZ [35] basis sets augmented by additional even-tempered basis functions. For the diatomics (and the carbon monoxide dimer), the aug-cc-pVTZ basis set was augmented by an additional set of diffuse functions (3s3p3d) located at the center of mass (of each monomer). The exponents for the first additional basis function for a given angular momentum were obtained as one half of the average of the exponents of the most diffuse basis functions with the same angular momentum in the original basis among atom types. For example, the exponent for the first $s$-type diffuse basis function for $\mathrm{CO}^{-}$ has been calculated as $\alpha_{1, s}=\frac{1}{2} \times \frac{1}{2}\left(\alpha_{d i f f, s}^{C}+\alpha_{d i f f, s}^{O}\right)=\frac{1}{2} \times \frac{1}{2}(0.04402+0.07376)$. Each subsequent exponent was obtained by division by two of the previous one: $\alpha_{i, l}=\alpha_{i-1, l} / 2$, where $l=s, p, d$. The CAP integrals were evaluated through numerical quadrature using a Becke-type grid of 99 radial points and 500 angular Levedev points. [36, 37].

For the model nitrogen-water cluster, the basis set and grid were chosen to replicate those used by Sommerfeld and Ehara [16] as closely as possible. On heavy atoms, the cc-pVTZ basis set was augmented with additional diffuse basis functions. For nitrogen, a (2s5p2d) set of even-tempered diffuse functions with scaling factors of $1 / 2$ for $\mathrm{s}$ and $\mathrm{d}$ functions, and $2 / 3$ for $\mathrm{p}$ basis functions was added. For oxygen, a set of (1s1p1d) diffuse functions with a scaling factor of $1 / 3$ was added. The grid used for computing the CAP integrals was a Becke-type grid of 500 radial points and 3470 angular Levedev points. [36, 37].

Bond lengths of 2.0740 and 2.1316 bohr were used for $N_{2}$ and $C O$, respectively. For the model nitrogen-water cluster, experimental equilibrium geometries were used for the monomers $\mathrm{N}_{2}$ and $\mathrm{H}_{2} \mathrm{O}$, while the $\mathrm{N}_{2}$-oxygen distance was varied, as indicated in Fig $1 \mathrm{~A}$. For the $C O$ dimer, a reference CCSD(T) geometry from Ref. [38] was chosen as the starting 
(A)

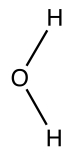

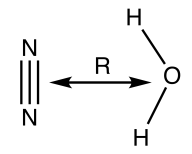

(B)

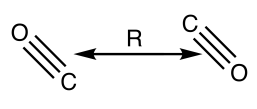

FIG. 1: Geometries of the $\mathrm{N}_{2}^{-}\left(\mathrm{H}_{2} \mathrm{O}\right)_{2}$ model cluster (A), and the $(\mathrm{CO})_{2}^{-}$dimer (B). Resonance states are investigated as functions of the intermolecular distance, $\mathrm{R}$.

point, and the distance between centers of mass of the two monomers was varied (Fig. 1B). Representative geometries of the clusters are given in the SI (Sec. SI).

\section{RESULTS AND DISCUSSION}

In this section, we compare computed resonance position and widths obtained from box and Voronoi CAPs for three types of systems: resonances in diatomic molecules (section III A), localized resonances in a model cluster (III B), and delocalized resonance states in a dimer (III C). In the discussion below, the box CAP size is specified by a single onset parameter $r_{b o x}$ in such a way that the onset in each dimension $\left(R_{\alpha}^{0}\right.$ in Eq. $\left.3, \alpha=x, y, z\right)$ is

obtained by adding $r_{b o x}$ to the maximum value of the nuclear coordinate in that dimension ( $\mathrm{x}, \mathrm{y}$, or $\mathrm{z})$. The box CAP is centered at the molecular center of mass. Voronoi CAPs are specified by the $r_{\text {cut }}$ cutoff radius, see Eq. 4 .

\section{A. Diatomics: $\mathrm{N}_{2}^{-}, \mathrm{CO}^{-}$}

The $\pi^{*}$ resonance states of $\mathrm{CO}^{-}$and $\mathrm{N}_{2}^{-}$arise from electron attachment to the lowest unoccupied molecular orbital (LUMO) of the neutral diatomic molecule. Figs. 2A,B show corrected (see Sec. II A) resonance energies and widths of the ${ }^{2} \Pi_{g}$ resonance of $N_{2}^{-}$, and the ${ }^{2} \Pi$ resonance of $\mathrm{CO}^{-}$, respectively, over a range of box sizes.

One can see that the effects of the CAP shape on the computed resonance parameters are rather minor for these two states (Fig. 2). The resonance energies and widths are slightly greater for the Voronoi CAP compared to those obtained with the box CAP. The widths are more sensitive to the shape of CAP than the resonance energies with the differences increasing with the increase of the CAP onsets. The resonance energies for the smallest onsets 
(A)
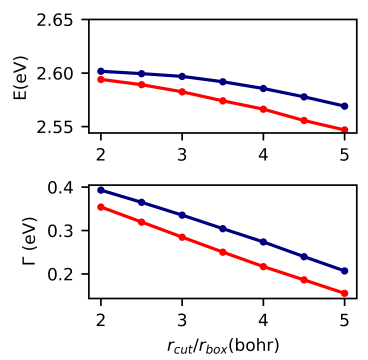

(B)
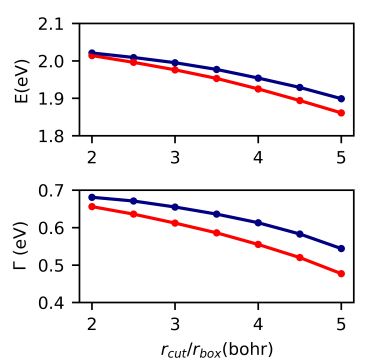

FIG. 2: Results for ${ }^{2} \Pi_{g}$ resonance of $N_{2}^{-}$(A) and for ${ }^{2} \Pi$ resonance of $C O^{-}$(B). Resonance energies are shown in the upper panels, and the widths are given in the lower panels. The values for box and Voronoi CAPs are shown in red and blue, respectively.

almost coincide in both cases, while for the largest values of $r_{c u t}$ and $r_{b o x}$, the differences between resonance energies computed with box and Voronoi CAP reach $0.02 \mathrm{eV}$ and $0.04 \mathrm{eV}$ for $\mathrm{N}_{2}^{-}$and $\mathrm{CO}^{-}$, respectively. Similarly, the largest deviations between the widths obtained with box and Voronoi CAPs are observed when $r_{c u t}$ and $r_{b o x}$ are set to 5 bohr $(0.05$ and $0.07 \mathrm{eV}$ for $\mathrm{N}_{2}^{-}$and $\mathrm{CO}^{-}$, respectively). Importantly, throughout the parameter range we explored, the influence of CAP shape on the results does not exceed that of the onset. The slightly greater resonance energy and width computed with Voronoi CAPs in comparison to those evaluated using analogous box CAPs can be explained by the Voronoi CAPs having an effectively smaller volume of the region where the CAP is zero, i.e. an effectively smaller $\mathrm{CAP}$ onset. Indeed, the values of resonance energies and widths decrease monotonously with the increase of the onset for both CAP types (Fig. 2). Lastly, the results are in good agreement with the numerous results which have been reported for these two states over time (see ref [26] and references therein).

To summarize, for the ${ }^{2} \Pi_{g}$ resonance of $N_{2}^{-}(\mathrm{A})$ and the ${ }^{2} \Pi$ resonance of $\mathrm{CO}^{-}$, the CAP type does not significantly affect the computed resonance parameters. Switching from a box to an analogous Voronoi CAP has the same effect on the results as simply reducing the size of the box. Therefore, we conclude that Voronoi and box CAPs perform similarly for small molecules, as the choice of CAP shape presents no unique challenges for these types of systems. 


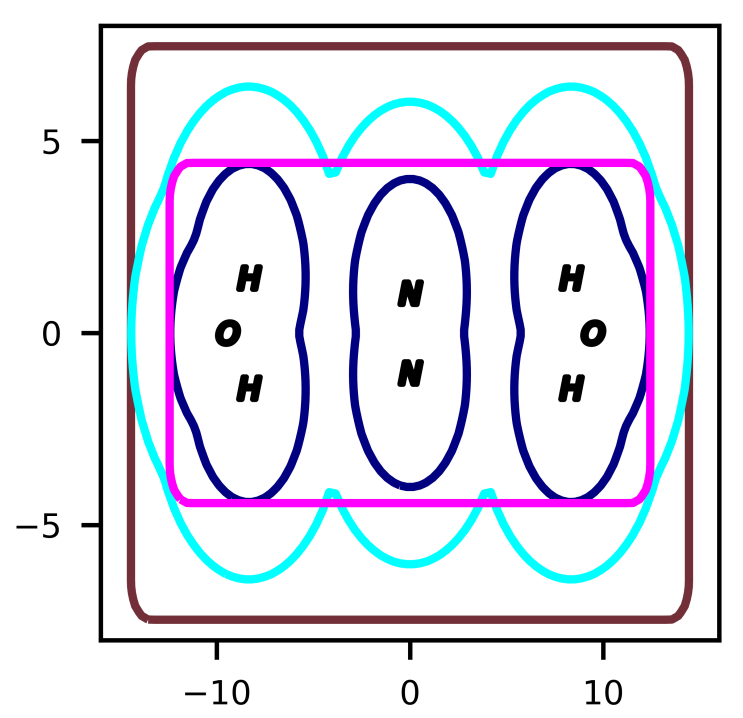

FIG. 3: Comparison of different box and Voronoi CAPs. Plotted is the isoline $\mathrm{W}=1$ hartree for box CAPs of 4 bohr (brown) and 2 bohr (magenta), and smooth Voronoi CAPs of 2 bohr (navy) and 4 bohr (cyan) in the plane of the molecular system.

\section{B. Localized resonance in a cluster: $\mathrm{N}_{2}^{-}\left(\mathrm{H}_{2} \mathrm{O}\right)_{2}$}

We turn our attention now to molecular clusters. A model $\mathrm{N}_{2}^{-}\left(\mathrm{H}_{2} \mathrm{O}\right)_{2}$ cluster was used as a pilot application of the Voronoi CAP by Sommerfeld and Ehara [16]. In this cluster, the two water molecules provide an environment which splits the ${ }^{2} \Pi_{g}$ resonance of $N_{2}^{-}$into in-plane $\left({ }^{2} B_{1 g}\right)$ and out-of-plane $\left({ }^{2} B_{3 g}\right)$ components [16]. In both states, the valence orbital corresponding to the outgoing electron remains primarily localized on the $N_{2}$ molecule. This model system serves as a test case to illustrate a key difference in the behavior of box and Voronoi CAPs. As shown in Fig. 3, when the water molecules are moved further from $N_{2}$, the Voronoi CAP will leak into the cavity between molecules. In contrast, the box CAP may instead exhibit regions of "dead space" [16]. Provided that this "dead space" volume is sufficiently large, the region may not be efficiently covered by the basis set, which can introduce artifacts into CAP calculations. To explore the effects of the CAP shape and size on the computed resonance parameters, we have considered three different onset values for Voronoi and box CAPs.

We computed complex potential energy curves (i.e. real and imaginary parts of the energy) as a function of water molecule distance $R$ (see Fig. 1A). Interaction with the water 
(A)
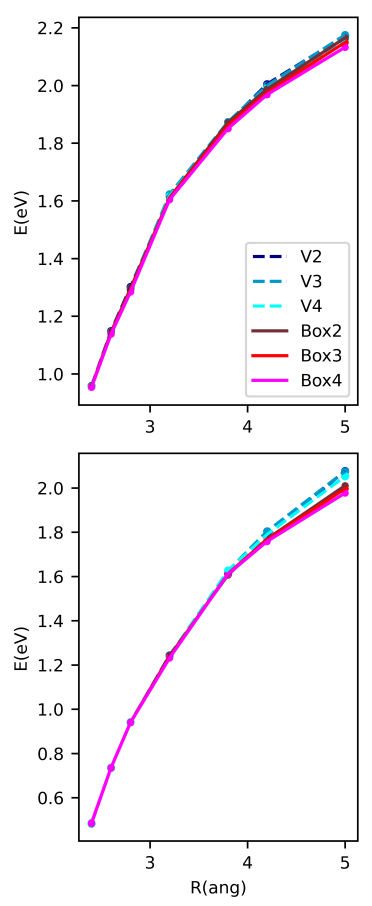

(B)
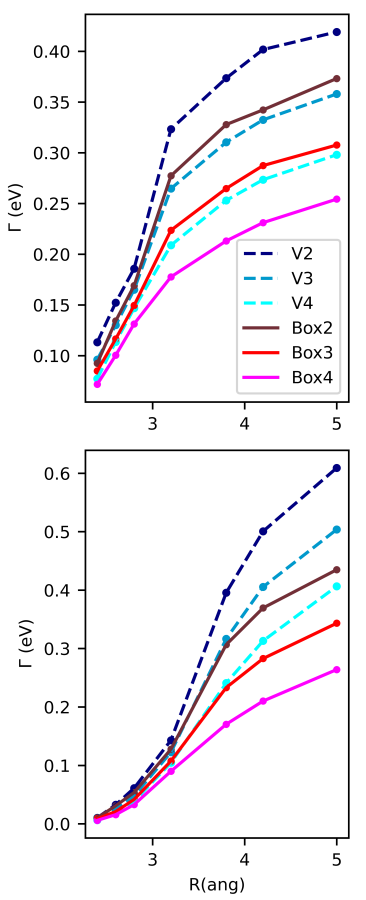

FIG. 4: Computed resonance positions (A) and widths (B) for the out-of-plane $B_{3 g}$ (upper panel) and in-plane $B_{1 g}$ (lower panel) resonance states of the model $\mathrm{N}_{2}^{-}\left(\mathrm{H}_{2} \mathrm{O}\right)_{2}$ cluster. The coordinate $\mathrm{R}$ on the abscissa is the distance between the center of the $N-N$ bond and the $O$ atoms on each water molecule. The labels "Box2", "Box3", and "Box4" denote $r_{b o x}$ values of 2 bohr, 3 bohr, and 4 bohr respectively for box CAPs. "V2", "V3", and "V4" denote $r_{c u t}$ values of 2, 3, and 4 bohr respectively for Voronoi CAPs.

molecules stabilizes both states, which is reflected in lower resonance energies and widths relative to the isolated $N_{2}^{-}$studied in the previous section. The environment has a larger effect on the in-plane component, lowering its energy and width relative to the out-of-plane component. For the out-of-plane $B_{3 g}$ state, the energies are insensitive to CAP choice or onset. The widths vary by at most $0.16 \mathrm{eV}$ between the smallest and largest CAPs for the range of $R$ values considered. The widths are consistently larger for Voronoi CAPs than box CAPs, similar to what we observed for the $N_{2}^{-}$monomer, which can be attributed to CAP size effect, i.e. Voronoi CAP being effectively tighter than box CAP with the same value of $r_{b o x}$. For the in-plane component, the dependence on the CAP type is more pronounced. The differences in resonance energies are still rather moderate: the largest deviation observed at 
$R=5 \stackrel{\circ}{A}$ is $0.10 \mathrm{eV}$ for different CAP types and sizes. Yet, the variance in the width is much greater than for the out-of-plane state; the results obtained with different CAP types vary by as much $0.35 \mathrm{eV}$. The differences between Voronoi and box CAPs with analogous onset values are also greater for the in-plane state, which can be explained by the Voronoi CAPs leaking into the intermolecular region along the $\mathrm{O}-\mathrm{N}-\mathrm{O}$ axis (Fig. 1A), making Voronoi CAP much tighter in comparison to the box one. Thus, the trends observed for the $\mathrm{N}_{2}-$ water dimer anion are similar to those for diatomic molecules and can be explained by variations in the CAP onset parameters.

Yet, we note that the trend we observed for the in-plane $B_{1 g}$ state differs from what was reported by Sommerfeld and Ehara [16]. While the results obtained using Voronoi CAPs were fairly similar, the authors in Ref [16] reported much larger widths when using the box CAP, which they attributed to artifacts caused by the presence of "dead space"; in other words reflections from the edge of the basis set. The discrepancy can be possibly attributed to the following causes. The first is the choice of the electronic structure method and specifics of CAP implementation. The CAP-augmented symmetry-adapted clusterconfiguration interaction (CAP/SAC-CI) method [15] used in Ref [16] employs a projected formulation in which the CAP is projected onto a subset of SAC-CI eigenvectors, while our CAP-EOM-EA-CCSD calculations include the CAP starting from Hartree-Fock [15, 26, 39] level. The second likely cause is the structure of the $\eta$-trajectories, which is discussed in further detail in the SI. Briefly, the box CAP $\eta$-trajectories contain a stationary region at smaller values of $\eta$ with noticeably lower energies and larger widths. The results reported here correspond to the stationary point at higher values of $\eta$ associated with a small plateau of $U^{R}$ and $U^{I}$ (Eq. 7)

Based on the reported data, the two CAP types behave comparably: the energies for both in-plane and out-of-plane states do not change much with the CAP shape or onset, while the widths obtained with Voronoi CAPs are larger than those computed using box CAP, similar to the trends observed for the isolated $N_{2}^{-}$. Importantly, the deviations in the computed resonance parameters for Voronoi and box CAP of comparable size are of similar magnitude to the deviations which occur from changing the CAP onset for a given CAP type. 


\section{Delocalized resonance: $(\mathrm{CO})_{2}^{-}$}

Finally, we examine the effect of CAP shape on delocalized resonance states. As a model system we use an anion of the carbon monoxide dimer. The neutral dimer has two stable isomers separated by $0.88 \mathrm{~cm}^{-1}$ that have been characterized both computationally and experimentally [29-31, 38]. Here, we report the first theoretical study of resonances in the carbon monoxide dimer. We explored the lower energy "a-state" isomer, in which the carbon atoms are arranged closer together in a slipped parallel geometry [29, 38]. Starting from a reference $\operatorname{CCSD}(\mathrm{T})$ geometry [38], we computed resonance parameters as a function of the distance $R$ (Fig. 1) between monomers for box and Voronoi CAPs of varying sizes.

The ${ }^{2} \Pi$ resonance in $\mathrm{CO}^{-}$splits into four states in the dimer, which arise from inphase/out-phase and in-plane/out-of-plane combinations of $\mathrm{CO} \pi^{*}$ orbitals. As shown in Fig. 5, the Dyson orbitals of the in-phase states posses significant amplitude in the space between the monomers, in contrast to the the out-of-phase states. Thus, we would expect the Voronoi CAP to noticeably perturb the in-phase states as it leaks into the cavity between monomers, while the out-of-phase states should not be affected as much.

Resonance energies for the out-of-phase states $\left({ }^{2} B_{g}\right.$ and $\left.{ }^{2} B_{u}\right)$ display the same trend of decreasing in energy as the monomers are stretched apart (Fig.6). As expected, the inphase out-of-plane ${ }^{2} A_{u}$ state is stabilized by the $\pi^{*}$ orbital overlap, and, therefore, its energy increases with intermolecular distance. Interestingly, the in-phase in-plane state ${ }^{2} A_{g}$ energy is lowered once the monomers are pulled apart. This counterintuitive result can possibly be attributed to either artifacts originating from the CAP or to stronger coupling to the continuum at larger values of $R$. As shown in Fig. 6, the effects of CAP shape and onset on resonance positions are much smaller for the out-of-phase states. For example, the ${ }^{2} B_{g}$ state shows very slight deviations between the results obtained from Voronoi and box CAPs; the differences are no greater than $0.05 \mathrm{eV}$. The resonance position of the in-phase ${ }^{2} A_{u}$ state, however, is very sensitive to the choice of CAP, with results that differ by as much as 0.23 $\mathrm{eV}$. The computed resonance positions for the in-phase states are systematically larger for Voronoi CAPs, with the trend becoming stronger as the system is stretched.

Turning our attention to the widths (Fig.7), the behaviors of the in-phase and out-ofphase states again differ. The effect of CAP shape is most pronounced for the in-phase ${ }^{2} A_{g}$ state. As the system is stretched, the widths obtained from Voronoi CAPs become 

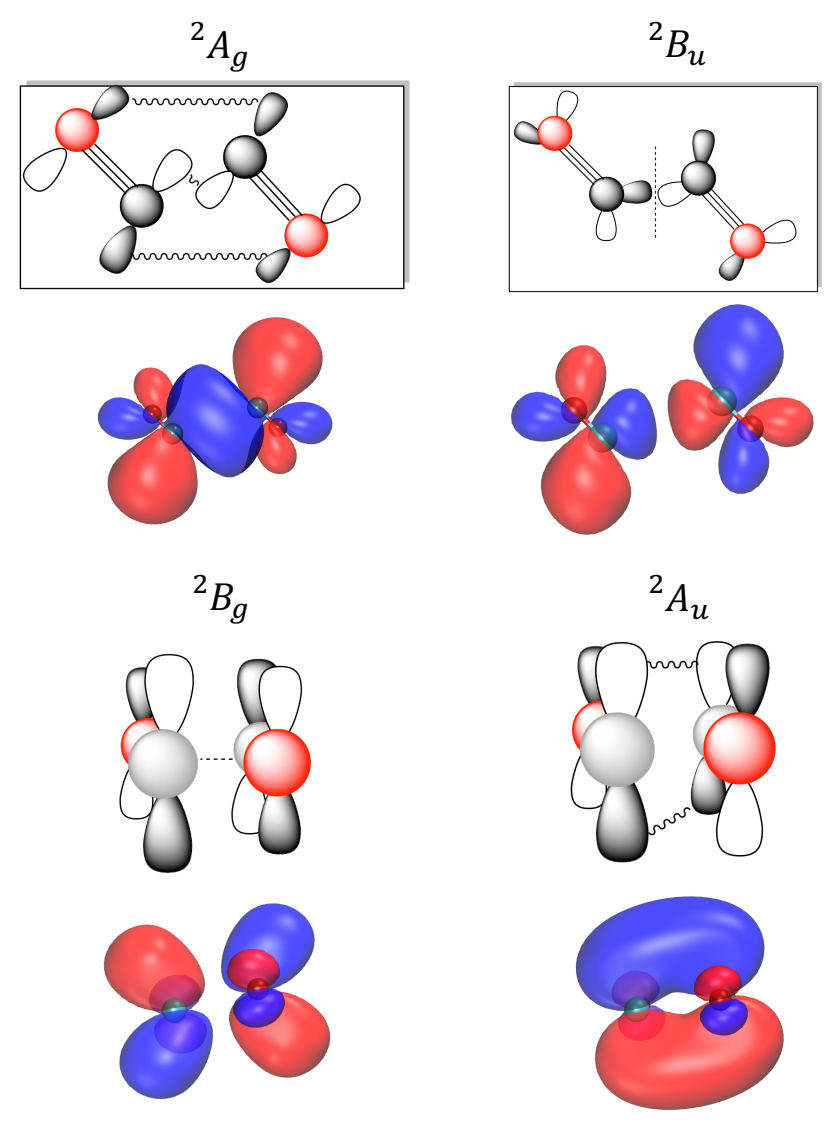

FIG. 5: Schematics and computed CAP-EOM-EA-CCSD Dyson orbitals for each of the four resonance states of the $(\mathrm{CO})_{2}^{-}$dimer. The Dyson orbitals were plotted at the equilibrium geometry, with an isosurface value of 0.02 at the minimum of the logarithmic velocity $\left|\eta \frac{\partial E}{\partial \eta}\right|$ for each state.

systematically larger than box CAPs, with the largest Voronoi CAP $\left(r_{c u t}=4\right.$ bohr) lying more than $0.1 \mathrm{eV}$ above the smallest box CAP $\left(r_{b o x}=2 \mathrm{bohr}\right)$ at the largest intermolecular distance. Importantly, for both in-phase states, the widths exhibit qualitatively different behavior when computed with Voronoi CAP in comparison to those with box CAP. The widths for the ${ }^{2} A_{g}$ state almost reach a plateau with box CAPs, while those obtained with Voronoi CAPs continue to rise sharply for large values of $R$. For the ${ }^{2} A_{u}$ state, the width exhibits a maximum for the two smaller Voronoi CAPs, whereas the width obtained with box CAPs monotonously rises. These inconsistencies between the box and Voronoi CAP can be possibly attributed to perturbations of the in-phase states caused by CAP leaking into the intermolecular space. Indeed, such differences are not present in the out-of-phase states, which as expected exhibit a relatively smaller influence of CAP shape on the computed 

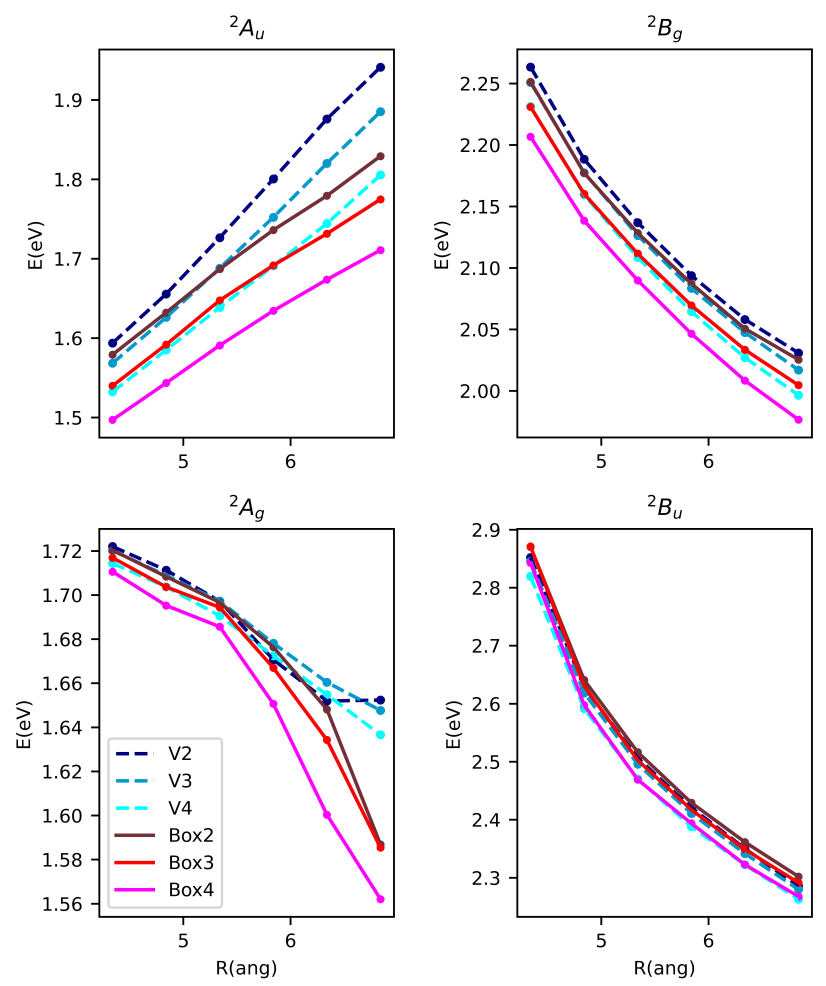

FIG. 6: Computed resonance positions for the four resonance states of the $(\mathrm{CO})_{2}^{-}$dimer. The coordinate $\mathrm{R}$ on the abscissa is the distance between centers of mass of the two $C O$ monomers (see Fig.1B). The labels "Box2", "Box3", and "Box4" denote box sizes of 2 bohr, 3 bohr, and 4 bohr respectively, while "V2", "V3", and "V4" denote cutoff radii of 2, 3, and 4 bohr respectively for Voronoi CAPs.

widths.

Overall, while Voronoi CAPs do not cause any notable artifacts for the out-of-phase states, which is consistent with less density in the intermolecular space for those states, greater deviations between the results obtained with box and Voronoi CAP are observed for the in-phase states. For the in-phase states, the Voronoi CAP leads to both higher widths and larger resonance energies, likely due to disruptions caused by the presence of CAP in the cavity of the stabilizing intermolecular interactions. 

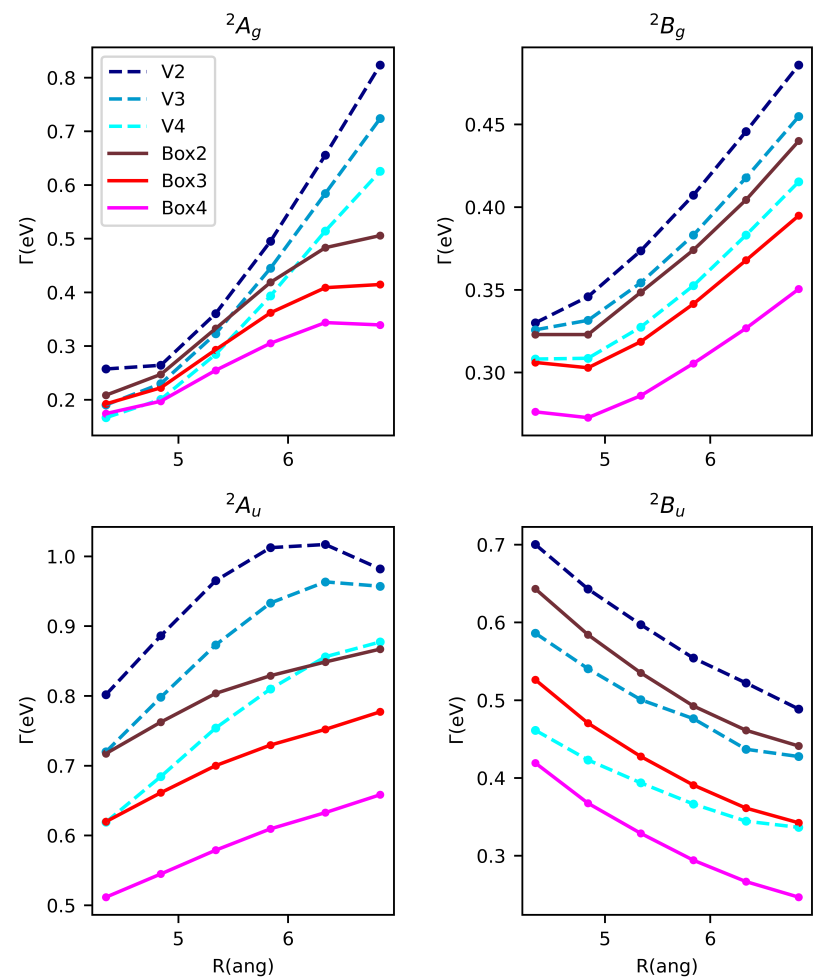

FIG. 7: Computed resonance widths for the four resonance states of the $(C O)_{2}^{-}$dimer. The coordinate $\mathrm{R}$ on the abscissa is the distance between centers of mass of the two $C O$ monomers (see Fig.1B). The labels "Box2", "Box3", and "Box4" denote box sizes of 2 bohr, 3 bohr, and 4 bohr respectively, while "V2", "V3", and "V4" denote cutoff radii of 2, 3, and 4 bohr respectively for Voronoi CAPs.

\section{CONCLUSIONS}

In summary, we have presented an implementation of the smooth Voronoi CAP for CAPEOM-CCSD methods, and analyzed its performance for three different types of systems. For diatomics, we observe very minor differences in resonance parameters obtained from box and Voronoi CAPs. The deviations observed for localized resonances in clusters can be explained by differences of the effective size of the CAPs. For delocalized resonances shared by two monomers, we observed the largest deviations for states with significant density in the intermolecular region, which can be attributed to the Voronoi CAP leaking into the intermolecular region and inducing perturbations. Finally, we note that while the Voronoi $\mathrm{CAP}$ is a natural choice for calculating resonance parameters for substantially changing molecular geometries, one should be careful about the nature of the state of interest to 
avoid overlap between CAP and the valence part of the resonance state. 


\section{ASSOCIATED CONTENT}

Supporting information. Geometries of molecular clusters, representative $\eta$ trajectories.

\section{ACKNOWLEDGMENTS}

This work was supported by National Science Foundation (CHE-1665276).

[1] Mukherjee, S.; Libisch, F.; Large, N.; Neumann, O.; Brown, L.; Cheng, J.; Lassiter, J.; Carter, E.; Norlander, P.; Halas, N. Hot electrons do impossible: plasmon-induced dissociation of $\mathrm{H}_{2}$ on Au. Nano Lett., 2013, 13, 240.

[2] Bressler, C.; Chergui, M. Ultrafast X-ray absorption spectroscopy. Chem. Rev., 2004, 104 , $1781-1812$.

[3] Corkum, P.; Krausz, F. Attosecond science. Nat. Phys., 2007, 3, 381-387.

[4] Strelkov, V. Role of autoionizing state in resonant high-order harmonic generation and attosecond pulse production. Phys. Rev. Lett., 2010, 104, 123901.

[5] Boudaïffa, B.; Cloutier, P.; Hunting, D.; Huels, M. A.; Sanche, L. Resonant formation of DNA strand breaks by low-energy (3 to $20 \mathrm{eV}$ ) electrons. Science, 2000, 287, 1658-1660.

[6] Millar, T. J.; Walsh, C.; Field, T. A. Negative Ions in Space. Chemical Reviews, 2017, 117(3), 1765-1795.

[7] Reinhardt, W. P. Complex coordinates in the theory of atomic and molecular structure and dynamics. Annu. Rev. Phys. Chem., 1982, 33, 223-255.

[8] Moiseyev, N. Non-Hermitian quantum mechanics. Cambridge University Press, 2011.

[9] Riss, U. V.; Meyer, H.-D. Calculation of resonance energies and widths using the complex absorbing potential method. J. Phys. B, 1993, 26, 4503-4536.

[10] Kosloff, R.; Kosloff, D. Absorbing boundaries for wave propagation problems. J. Comput. Phys., 1986, 63(2), 363-376.

[11] Sahoo, S.; Ho, Y. K. The complex absorbing potential method (CAP) to study the stark effect in hydrogen and lithium. Journal of Physics B: Atomic, Molecular and Optical Physics, jun 
2000, 33(12), 2195-2206.

[12] Muga, J.; Palao, J.; Navarro, B.; Egusquiza, I. Complex absorbing potentials. Physics Reports, 2004, 395(6), $357-426$.

[13] Zhang, L.; Chen, J.; Wang, J. First-principles investigation of transient current in molecular devices by using complex absorbing potentials. Phys. Rev. B, May 2013, 87, 205401.

[14] Jagau, T.; Bravaya, K.; Krylov, A. Extending quantum chemistry of bound states to electronic resonances. Annu. Rev. Phys. Chem., 2017, 68, 525-553.

[15] Ehara, M.; Sommerfeld, T. CAP/SAC-CI method for calculating resonance states of metastable anions. Chem. Phys. Lett., 2012, 537, 107-112.

[16] Sommerfeld, T.; Ehara, M. Complex Absorbing Potentials with Voronoi Isosurfaces Wrapping Perfectly around Molecules. Journal of Chemical Theory and Computation, 2015, 11(10), 4627-4633.

[17] Ghosh, A.; Vaval, N.; Pal, S. Equation-of-motion coupled-cluster method for the study of shape resonances. J. Chem. Phys., 2012, 136, 234110.

[18] Phung, Q. M.; Komori, Y.; Yanai, T.; Sommerfeld, T.; Ehara, M. Combination of a voronoitype complex absorbing potential with the xms-caspt2 method and pilot applications. $J$. Chem. Theory Comput., 2020, 16, 2606-2616.

[19] Kunitsa, A.; Granovsky, A.; Bravaya, K. CAP-XMCQDPT2 method for metastable electronic states. J. Chem. Phys., 2017, 146, 184107.

[20] Santra, R.; Cederbaum, L. Complex absorbing potentials in the framework of electron propagator theory. I. General formalism. J. Chem. Phys., 2002, 117, 5511-5521.

[21] Sajeev, Y.; Sindelka, M.; Moiseyev, N. Reflection-free complex absorbing potential for electronic structure calculations: Feshbach type autoionization of helium. Chem. Phys., 2006, 329, 307-312.

[22] Sajeev, Y.; Moiseyev, N. Reflection-free complex absorbing potential for electronic structure calculations: Feshbach-type autoionization resonances of molecules. J. Chem. Phys., 2007, $127,034105$.

[23] Riss, U.; Meyer, H.-D. The transformative complex absorbing potential method: a bridge between complex absorbing potentials and smooth exterior scaling. J. Phys. B, 1998, 31, 2279-2304.

[24] Santra, R.; Cederbaum, L.; Meyer, H.-D. Electronic decay of molecular clusters: non- 
stationary states computed by standard quantum chemistry methods. Chemical Physics Letters, 1999, 303(3), $413-419$.

[25] Jagau, T.-C.; Zuev, D.; Bravaya, K.; Epifanovsky, E.; Krylov, A. A fresh look at resonances and complex absorbing potentials: Density matrix based approach. J. Phys. Chem. Lett., 2014, 5, 310-315.

[26] Zuev, D.; Jagau, T.-C.; Bravaya, K. B.; Epifanovsky, E.; Shao, Y.; Sundstrom, E.; HeadGordon, M.; Krylov, A. Complex absorbing potentials within EOM-CC family of methods: Theory, implementation, and benchmarks. J. Chem. Phys., 2014, 141, 024102.

[27] Kanazawa, Y.; Ehara, M.; Sommerfeld, T. Low-lying $\pi^{*}$ resonances of standard and rare dna and rna bases studied by the projected CAP/SAC-CI method. The Journal of Physical Chemistry A, 2016, 120(9), 1545-1553. PMID: 26878328.

[28] Shao, Y.; Gan, Z.; Epifanovsky, E.; Gilbert, A.T.B.; Wormit, M.; Kussmann, J.; Lange, A.W.; Behn, A.; Deng, J.; Feng, X., et al. Advances in molecular quantum chemistry contained in the Q-Chem 4 program package. Mol. Phys., 2015, 113, 184-215.

[29] Rezaei, M.; Sheybani-Deloui, S.; Moazzen-Ahmadi, N.; Michaelian, K. H.; McKellar, A. R. W. CO Dimer: The Infrared Spectrum Revisited. The Journal of Physical Chemistry A, 2013, $117(39), 9612-9620$.

[30] Brookes, M. D.; McKellar, A. The mystery of the CO dimer: assignments from variabletemperature jet-cooled infrared spectra. Chem. Phys. Lett., 1998, 287, $365-370$.

[31] Brookes, M. D.; McKellar, A. R. W. Infrared spectrum and energy levels of the CO dimer: Evidence for two almost isoenergetic isomers. J. Chem. Phys., 1999, 111, 7321-7328.

[32] Moiseyev, N. Quantum theory of resonances: calculating energies, widths and cross-sections by complex scaling. Phys. Rep., 1998, 302, 212-293.

[33] Santra, R.; Cederbaum, L. Non-hermitian electronic theory and applications to clusters. Phys. Rep., 2002, 368, 1-117.

[34] Dunning, T. Gaussian basis sets for use in correlated molecular calculations. I. The atoms boron through neon and hydrogen. J. Chem. Phys., 1989, 90, 1007-1023.

[35] Kendall, R.; T.H. Dunning, J.; Harrison, R. Electron affinities of the first-row atoms revisited. systematic basis sets and wavefunctions. J. Chem. Phys., 1992, 96, 6796-6806.

[36] Becke, A. D. A multicenter numerical integration scheme for polyatomic molecules. J. Chem. Phys., 1988, 88, 2547-2553. 
[37] Lebedev, V. Values of the nodes and weights of ninth to seventeenth order Gauss-Markov quadrature formulae invariant under the octahedron group with inversion. Zh. Vychisl. Mat. Mat. Fiz., 1975, 15, 48-54.

[38] Vissers, G. W. M.; Heßelmann, A.; Jansen, G.; Wormer, P. E. S.; van der Avoird, A. New $\mathrm{CO}-\mathrm{CO}$ interaction potential tested by rovibrational calculations. The Journal of Chemical Physics, jan 2005, 122(5), 54306.

[39] Sommerfeld, T.; Santra, R. Efficient method to perform CAP/CI calculations for temporary anions. Int. J. Quant. Chem., 2001, 82, 218-226. 